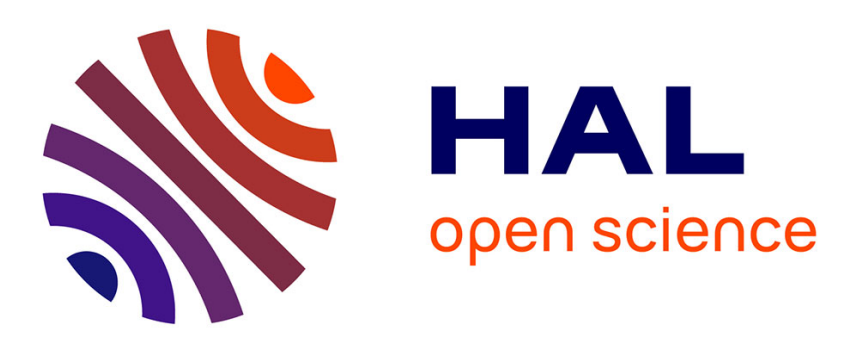

\title{
Precise force controls enhance loudness discrimination of self-generated sound
}

\author{
Nozomi Endo, Takayuki Ito, Takemi Mochida, Tetsuya Ijiri, Katsumi \\ Watanabe, Kimitaka Nakazawa
}

\section{- To cite this version:}

Nozomi Endo, Takayuki Ito, Takemi Mochida, Tetsuya Ijiri, Katsumi Watanabe, et al.. Precise force controls enhance loudness discrimination of self-generated sound. Experimental Brain Research, 2021, 239 (4), pp.1141-1149. 10.1007/s00221-020-05993-7 . hal-03014888

\section{HAL Id: hal-03014888 \\ https://hal.science/hal-03014888}

Submitted on 8 Dec 2021

HAL is a multi-disciplinary open access archive for the deposit and dissemination of scientific research documents, whether they are published or not. The documents may come from teaching and research institutions in France or abroad, or from public or private research centers.
L'archive ouverte pluridisciplinaire HAL, est destinée au dépôt et à la diffusion de documents scientifiques de niveau recherche, publiés ou non, émanant des établissements d'enseignement et de recherche français ou étrangers, des laboratoires publics ou privés. 
1 Precise force controls enhance loudness discrimination of self-generated sound

2

3 Nozomi Endo ${ }^{1,5,6}$, Takayuki Ito ${ }^{2,3}$, Takemi Mochida ${ }^{4}$, Tetsuya Ijiri ${ }^{1}$, Katsumi Watanabe ${ }^{5}$,

$4 \quad$ Kimitaka Nakazawa ${ }^{1}$

5

$6{ }^{1}$ Graduate School of Arts and Sciences, The University of Tokyo, 3-8-1, Komaba,

7 Meguro-ku, Tokyo 153-8902, Japan

$8{ }^{2}$ Univ. Grenoble Alps, Grenoble-INP, CNRS, GIPSA-lab, 11 rue des Mathématiques,

9 Grenoble Campus BP46, F-38402 SAINT MARTIN D'HERES CEDEX, France

$10 \quad{ }^{3}$ Haskins laboratories, 300 George Street New Haven, CT 06511, USA

11

${ }^{4}$ NTT Communication Science Laboratories, 3-1, MorinosatoWakamiya Atsugi-shi, Kanagawa 243-0198, Japan

13

14

${ }^{5}$ Faculty of Science and Engineering, Waseda University, 3-4-1, Ohkubo, Shinjuku-ku, Tokyo 169-8555, Japan

${ }^{6}$ Japan Society for the Promotion of Science, Chiyoda-ku, Tokyo 102-0083, Japan 
18 Acknowledgements. This study was partially supported by research grants from JSPS

19 KAKENHI Grant Number 20J14743 awarded to N.E.; the National Institute on

20 Deafness and Other Communication Disorders Grant R01-DC017439 awarded to T.I.;

21 JSPS KAKENHI Grant Numbers $17 \mathrm{H} 00753$ and 17H06344 awarded to K.W.; JST

22 CREST JPMJCR14E4 and JPMJCR16E1 awarded to K.W. and K.N.; JSPS KAKENHI

23 Grant Number $18 \mathrm{H} 04082$ awarded to K.N. 
Motor executions alter sensory processes. Studies have shown that loudness perception

26 changes when a sound is generated by active movement. However, it is still unknown

27 where and how the motor-related changes in loudness perception depend on the task

28 demand of motor execution. We examined whether different levels of precision

29 demands in motor control affects loudness perception. We carried out a loudness

30 discrimination test, in which the sound stimulus was produced in conjunction with the

31 force generation task. We tested three target force amplitude levels. The force target was

32 presented on a monitor as a fixed visual target. The generated force was also presented

33 on the same monitor as a movement of the visual cursor. Participants adjusted their

34 force amplitude in a predetermined range without overshooting using these visual

35 targets and moving cursor. In the control condition, the sound and visual stimuli were

36 generated externally (without a force generation task). We found that the discrimination

37 performance was significantly improved when the sound was produced by the force

38 generation task compared to the control condition, in which the sound was produced

39 externally, although we did not find that this improvement in discrimination

40 performance changed depending on the different target force amplitude levels. The 
41 results suggest that the demand for precise control to produce a fixed amount of force may be key to obtaining the facilitatory effect of motor execution in auditory processes.

44 Keywords: auditory perception, auditory-motor interaction, motor execution,

45 self-generated sound

46

47 

Auditory-motor interaction is important in speech and musical performance

50 (Guenther et al. 1998; Zatorre et al. 2007). In both performances, movement needs to be precisely controlled to generate a target sound, and motor performances are affected by auditory inputs (Pfordresher and Palmer 2006; Houde and Jordan 1998). In addition to auditory processing influencing motor performance, motor performance also influences auditory processing. The effect of motor execution in auditory processing has been investigated in the context of sensory attenuation of self-generated sounds. Studies involving magnetoencephalography show that the amplitude of auditory event-related responses was attenuated by self-generated sounds compared to externally generated sounds (Aliu et al. 2009; Baess et al. 2011; Martikainen et al. 2005). Indeed, the attenuation of auditory event-related responses has been reported in the processing of speech sounds

61 (Niziolek et al. 2013; Tian and Poeppel, 2010). It is hypothesized that the auditory attenuation that occurs due to motor execution contributes to the distinction between

63 self-produced sensation and sensation due to external reasons by using 'efference copy' or 'corollary discharge' (i.e., a copy of motor command sent from the motor areas to the sensory areas; Wolpert et al. 1995; Wolpert and Flanagan 2001). In the postulated model, 
motor commands are sent to the executing effectors for voluntary movement, and in

67 parallel, an efference copy is sent to the relevant sensory area where sensory signals

68 consequential to the voluntary movement are expected to be received. This copy helps

69 to distinguish between self-generated and externally generated sounds.

70 Motor executions change the perception of sounds. In speech sound processing,

71 motor execution biases speech perception towards the sound expected from produced

72 speech movements (Sams et al. 2005; Mochida et al. 2013). Niziolek et al. (2013)

73 showed that the attenuation of auditory cortical responses due to participants' speaking

74 was reduced when the generated sound was less prototypical.

The change by motor executions has also been reported in non-speech producing tasks, such as finger movements. Self-generated sounds in a button press task are perceived as less loud compared to externally generated sounds (Sato 2008, Weiss et al. 2011). Reznik et al. (2014) showed that binaural hearing thresholds are lower for self-generated sounds at the near-detection threshold level. Regarding the interaction between the control of finger force and loudness perception, we empirically acknowledge that applying a stronger force while tapping on a desk or even on a piano key generates a louder sound (Kinoshita et al. 2007). Kunde

83 (2001) found that varying force affects the process of the manual choice reaction task of 
84 loudness perception. This suggests that precise control of the produced force in motor

85 execution may play a role in loudness perception. However, this point is still unclear

86 because the button press task applied in the previous studies does not require precise

87 control of the amplitude of the produced force as long as a button was pressed at a

88 certain level. On the other hand, auditory attenuation can fluctuate depending on

89 movement variability. Neszmélyi and Horváth (2017) demonstrated that auditory

90 event-related potentials in response to self-generated sounds were different among

91 strong and soft pinch fluctuations. This suggests that motor influences on auditory

92 perception may depend on the task context, not simply on the presence or absence of

93 motor execution, but in the ways in which movements (and required force generations)

94 are executed. We thus expect that an explicit control of the produced force in a finger

95 movement for sound production may change loudness perception through

96 auditory-motor interaction.

97 The current study aimed to examine whether self-generated sounds produced with

98 the adjustment of force amplitude can be processed differently from externally

99 generated sounds and to determine whether the level of precision required by a task

100 affects loudness perception. We carried out experiments using loudness discrimination

test with and without a force generation task (Motor and Non-Motor conditions). In 
102 Motor condition, the sound stimulus was produced when the produced force reached the

103 imposed target amplitude. The produced force was presented using a moving cursor, and

104 the target force was presented as a fixed horizontal bar. The participant's task was to

105 adjust the produced force to the target with the visual targets and moving cursor. We

106 tested three different target forces. In Non-Motor condition, the sound was produced

107 externally. We estimated the point of subjective equality (PSE) as a measure of

108 perceptual bias and just noticeable difference (JND) as a measure of discrimination

109 performance. Our prediction was that there would be changes in perceptual bias and/or

110 discrimination performance due to the presence/absence of the force generation task and

111 the amplitude of the target force.

112

\section{EXPERIMENT 1}

\section{Methods}

\section{Participants}

118 Twenty-five healthy students (mean age $=22.1, \mathrm{SD}=2.5$ years) participated in

119 Experiment 1. The participants were all right-handed with normal hearing and were 
naïve to the purpose of the experiment. The experimental protocols were in accordance

121 with the guidelines set out in the Declaration of Helsinki. All participants signed an

122 informed consent form approved by the Waseda University ethics board.

123

124

125

126

127

128

129

130

131

132

133

134

135

136

\section{Apparatus, stimuli, and procedure}

The participants were seated in front of a monitor (EV2450, EIZO) with headphones (HD280, Sennheiser). The right hand was gently placed on a desk or a force sensor (USL06-H5-50N-D-FZ, Tec Gihan), and the left hand was placed on a keyboard.

The surface of the force sensor was set horizontally on the desk. The force signals from the sensor were amplified and filtered with a 5-Hz low-pass filter in the amplifier (DSA-03A, Tec Gihan). The filtered signals were transferred to a laptop computer via an analog-to-digital converter (NI 9215, National Instruments) with a sampling frequency of $200 \mathrm{~Hz}$. Data acquisition and stimulus presentation were carried out using MATLAB (MathWorks, Inc.) with the Data Acquisition Toolbox (MathWorks, Inc.) and Psychophysics Toolbox extensions (Brainard 1997;Pelli 1997; Kleiner et al. 2007). Loudness discrimination test: Two 1000-Hz sine tones (Fig. 1A) were binaurally presented for $250 \mathrm{~ms}$, separated by an interval of $1000 \mathrm{~ms}$, through the headphones. The participants were asked to indicate whether the second sound was louder or softer than the first sound by pressing the keys on the keyboard with their left hand. The amplitude 
138 of the first sound was fixed at $65 \mathrm{~dB}$ in all trials (standard stimulus), and the amplitude

139 of the second sound was varied in each trial (comparison stimulus). We used seven

140 levels of loudness, from $62 \mathrm{~dB}$ to $68 \mathrm{~dB}$, in 1-dB increments. The reaction time between

141 the end of the second stimulus and the participant's response was recorded.

$142 \quad$ Force generation task for sound presentation: To generate the sound stimulus, the

143 participants were asked to perform a force generation task, in which they produced a

144 specific amplitude of the force by pressing the force sensor with their right index finger.

145 They were instructed to complete the entire movement to reach the target amplitude and

146 then to release the force in a short period of time (approx. $500 \mathrm{~ms}$ ). The index finger

147 was positioned slightly above the sensor without touching the start, and returned to the

148 original position at the end of the trial so that the force was completely removed from

149 the sensor at the start and end of each trial. The amplitude of the produced force was

150 reflected in real-time on the monitor as a vertical movement of a cross cursor mark (Fig.

151 1B). The task was to make the cursor reach the target height presented on the same

152 monitor using a horizontal bar. We also showed the upper limit of the production force

153 (0.5 N larger than the target level) using an additional horizontal bar (Fig. 1B) in order

154 to prevent participants from producing a force level large enough to cover all target amplitudes. The sound stimulus was produced when the peak amplitude of the force was 
within the range of the target and the upper limit of the force. The participants practiced

157 before the main experiment, based on the experimenter's instructions, as described

158 below. When the force was produced over the upper limit, the trial was excluded and

159 redone in the following trial.

160 Experimental procedure: We carried out the loudness discrimination test using two

161 conditions: with the force generation task (Motor condition) and without the task

162 (Non-Motor condition).

163 In Motor condition, the force generation task was involved in the presentation of

164 the standard stimulus, but not of the comparison stimulus. We tested three levels of

165 target force $(1 \mathrm{~N}, 2 \mathrm{~N}$, and $4 \mathrm{~N})$. In visual instruction, the cursor position was

166 proportional to the change in the force, and hence the height of the target level varied

167 depending on the target force (Fig. 1B).

168 In Non-Motor condition, participants placed their right hand gently on the desk

169 without any force generation or movement. We replayed the force data recorded in

170 Motor condition to replicate the cursor movement. The standard stimulus was played

171 when the cursor reached the target level on the monitor, as in Motor condition. For this

172 reason, Non-Motor condition was carried out after at least one session of Motor condition was performed. 
175 Non-Motor conditions), with a 5-minute interval between sessions. In one session, 21

176 combinations (three target forces $\times$ seven loudness levels) with eight repetitions were

177 tested in random order (168 trials in total). We carried out two types of session order: 1)

178 Motor condition and Non-Motor condition were alternately tested (alternated pattern),

179 or 2) Motor condition was repeated twice, and then Non-Motor condition was repeated

180 twice (separated pattern). These session orders were counterbalanced among the

181 participants.

182 Two practice sessions were carried out before the main test to habituate the

183 participants to the force generation task in Motor condition. In these two sessions, the

184 participants practiced using only one level of target force in three levels $(1 \mathrm{~N}, 2 \mathrm{~N}$, or 4

$185 \mathrm{~N})$ for simplification. The number of participants assigned to each level was

186 approximately equal. The first practice session was to practice performing the force

187 generation performance with the visual target in order to produce the standard stimulus

188 described above. In the main test, the standard stimulus was presented when the

189 generated force reached the target amplitude, but not the comparison stimulus. The

190 practice was repeated 30 times. The experimenter verbally instructed and corrected the 
192 discrimination task with the practiced force generation task. The two extreme levels of 193 sound stimulus (62 $\mathrm{dB}$ and $68 \mathrm{~dB})$ were used as an example for the comparison stimulus.

194 The participants were asked whether the first or second sound stimulus was louder. Five 195 repetitions in each pair (ten trials in total) were performed in random order. The 196 experimenter verified whether the participants carried out the task and responded 197 correctly.

\section{Data analysis}

200 We calculated the probability that the participants perceived the second sound as 201 being louder for each comparison stimulus. Psychometric functions were obtained by 202 fitting cumulative Gaussians using a maximum-likelihood procedure in Motor and 203 Non-Motor conditions separately. Based on the fitted psychometric curve, we obtained 204 the PSE and the JND. PSE represents the loudness level in which $50 \%$ of trials are 205 perceived as louder than the standard. When a participant perceives the first sound as 206 being louder than the second sound, the PSE value becomes larger. If the force 207 generation task in Motor condition modifies the loudness perception of the first sound, 208 the PSE in Motor condition can be changed from that in Non-Motor condition as perceptual bias. JND represents the minimum difference in amplitude due to which a 
210 participant is able to discriminate between the standard and comparison stimuli. JND is

211 defined as half the difference of the comparison tone magnitude judged as louder by

$21275 \%$ and judged as louder on $25 \%$ of trials. When the JND value is small, a participant

213 is better able to discriminate between the two sounds. In addition to PSE, JND is also

214 expected to be modified between Motor and Non-Motor conditions. We took averages

215 for each condition on a per-participant basis for the reaction times. PSE, JND, and

216 reaction time were analyzed using a two-way ANOVA with motor factor (Motor

217 condition/ Non-Motor condition) and force factor $(1 \mathrm{~N}, 2 \mathrm{~N}$, and $4 \mathrm{~N})$ as

218 within-participant factors. For post-hoc tests, we applied a simple main effect test when

219 the interaction was observed and applied a Bonferroni correction for the significant

220 main effect of force factor, which had three conditions.

221

222 Results

223 Fig. 2A shows the JND values for each motor condition across the three task force

224 amplitudes. In JND, the main effect of motor factor was significant $(F(1,24)=16.20$,

$\left.225 p=0.0005 ; \eta p^{2}=0.40\right)$. In addition, the JND values in Motor condition were smaller

226 than those in Non-Motor condition. We also found a significant main effect of force

227 factor $\left(F(2,48)=4.00, p=0.0248, \eta p^{2}=0.14\right)$. The JND values significantly increased 
as the target force increased. Post-hoc comparisons with Bonferroni correction showed that JNDs in the 4-N condition were significantly larger than in the $1-\mathrm{N}(p=0.0104)$ and $2-\mathrm{N}(p=0.0371)$ conditions. There was no significant interaction between motor factor and force factor $(F(2,48)=0.82, p=0.4481)$. In the PSE values (Fig. 2B), we did not

232 find significant differences in all factors: the main effect of motor factor $(F(1,24)=$ 233 2.06, $p=0.1640)$, force factor $(F(2,48)=0.51, p=0.6060)$, and two-way interaction $234 \quad(F(2,48)=0.07, p=0.9367)$. ANOVA by adding the factor of session order (alternated pattern and separated pattern) as between-subject factors into the model used in the main analysis. The results showed that the session order factor was not significant $(F(1,23)=0.92, p=0.3469)$, while the main significant effect between Motor and Non-Motor conditions $(F(1,23)=17.97, p=$ 0.0003, $\left.\eta p^{2}=0.87\right)$ and force factor $\left(F(2,46)=3.91, p=0.0270, \eta p^{2}=0.18\right)$ were maintained. There was also a significant interaction between the session order factor and the motor factor $\left(F(1,23)=4.78, p=0.0392, \eta p^{2}=0.21\right)$. In the post-hoc test, the simple-main effect test showed a significant effect of motor factor in the separated

244 pattern group $\left(\mathrm{F}(1,23)=20.65, \mathrm{p}=0.0001, \eta \mathrm{p}^{2}=0.90\right)$, but not in alternated pattern $\operatorname{group}(F(1,23)=2.106, p=0.1603)$ 
248 difference $(\mathrm{F}(1,24)=0.28, \mathrm{p}=0.6000)$ between them. Further, we did not find any significant differences regarding the main effect of force factor $(\mathrm{F}(2,48)=0.17, \mathrm{p}=$ 250 0.8476)or the two-way interaction $(F(2,48)=1.53, \mathrm{p}=0.2284)$.

252 the sound was generated by the motor task. We also found that loudness discrimination was improved with a small amplitude of the target force, irrespective of the motor factor.

254 No statistical evidence for differences in perceptual bias was observed. We found no reliable differences in the reaction time.

\section{Discussion}

The overall goal of the present study was to examine whether the amplitude of the force produced changes the perceived loudness and discriminability of sounds.

260 Experiment 1 tested this idea using a specific visual situation, in which three levels of target force were presented visually at different heights. We found that generating sound in conjunction with motor execution improved loudness discrimination, but did not bias

263 loudness perception. This finding is consistent with previous studies reporting 
264 improvement in perceptual decisions for self-generated stimuli (Reznik et al., 2014;

265 Myers et al., 2020).

266 We also found that the discrimination performance was better with smaller target

267 forces. However, the change was similarly observed in both Motor and Non-Motor

268 conditions; therefore, this might not be attributed to the actual force produced in the

269 force generation task. The target forces were represented as three different heights on

270 the monitor in both Motor and Non-Motor conditions. This contextual information

271 based on height might cause similar changes in the two conditions. Thus, we considered

272 this possibility in the next experiment using the same height for the three target force

273 conditions.

274

275

276

277

EXPERIMENT 2

278

We examined whether the observed effect of the target force in Experiment 1 was

279

induced indifferent visual instructions of the target force, in which the height of the

280

visual target was equated. 


\section{Participants}

Twenty-five healthy right-handed students with normal hearing participated in the

284 experiment $($ mean age $=21.5, \mathrm{SD}=3.1$ years $)$ and none participated in Experiment 1.

285 All participants were naïve to the purpose of the experiment. The experimental 286 protocols were in accordance with the guidelines set forth in the Declaration of Helsinki 287 and were approved by the Waseda University ethics board. All participants signed 288 approved informed consent forms.

289

290 Methods

291 The experimental procedure was the same as in Experiment 1, except for the visual

292 presentation used in the force generation task. We applied the same height for all the 293 target force levels (Fig. 1C), resulting in the velocity of cursor movement, given that the 294 produced force varied depending on the target force condition.

\section{$296 \quad$ Results}

Fig. 3A shows the JND values in Experiment 2. In JND, we found that the main

298 effect of motor factor was significant $\left(F(1,24)=11.37, p=0.0025 ; \eta p^{2}=0.32\right)$. The 
in Experiment 1. Contrary to the results obtained in Experiment 1, the main effect in the

301 force factor was not significant $(F(2,48)=0.35, p=0.7066)$. We also did not find any

302 interaction effect $(F(2,48)=0.30, p=0.7413)$. For PSE values, we did not find any

303 significant difference (Fig. 3B) regarding the main effect of motor factor $(F(1,24)=$

$3040.77, p=0.3865)$, force factor $(F(2,48)=0.26, p=0.7699)$, and the two-way

305 interaction $(F(2,48)=0.99, p=0.3801)$.

306 For the effect of session order in JND values, we carried out three-way ANOVA by

307 including the session order as in Experiment 1. The results showed that there was no

308 significant effect in the session order factor $(F(1,23)=0.05, p=0.8314)$, while the

309 main effect between Motor and Non-Motor conditions was maintained. The interaction

310 between the session order factor and the motor factor was not significant $(F(1,23)=$

$311 \quad 0.64, \mathrm{p}=0.4338)$.

312 The reaction time averaged across participants in Motor condition (732 $\mathrm{ms} \pm 105$

313 s.e.) was shorter than that in Mon-Motor condition ( $802 \mathrm{~ms} \pm 184$ s.e.). The main effect

314 of motor factor was significant $\left(F(1,24)=5.35, p=0.0296, \eta p^{2}=0.05\right)$, while the force

315 factor $(F(2,48)=0.06, p=0.9398)$ and two-way interaction $(F(2,48)=1.49, p=0.2355)$

316 were not significant. 

in Experiment 1 and Experiment 2. The discrimination performance was better in the

319 force generation task in both experiments. On the other hand, we also found differences in two experiments. We did not find any change in discrimination performance across

321 the target force levels and any difference by session order. Reaction time was different 322 between Motor and Non-Motor conditions, which was not observed in Experiment 1.

\section{Discussion}

In Experiment 2, despite the fact that the target height was constant and that the cursor movement velocity was changed, we found consistent results of auditory

327 accuracy between Motor and Non-Motor conditions with those obtained in Experiment

328 1. The sound generated in conjunction with motor execution improved the performance 329 of loudness discrimination without influencing loudness perception. Unlike Experiment 330 1, we did not observe modulation of the discrimination performance based on the target 331 force amplitudes. We also obtained different results from Experiment 1 in reaction time and in session order effect. These differences between these two experiments might be due to differences in visual instruction. 
We found that discrimination performance was significantly improved when the sound stimulus was produced with the force generation task compared to when the sound stimulus was produced externally, whereas perceptual bias did not change

339 between with and without the force generation task. Since the results were consistent in

340 all three force target conditions and different visual instructions between Experiment 1

341 and Experiment 2, the task requirement to produce a specific amount of finger force,

342 compared to a simple button pressing action without any requirement of force control,

343 can play an important role in enhancing loudness discrimination. Although we expected

344 that this enhancement would change depending on the target of force production, we did

345 not find the expected tendency in the current experimental situation.

346 In addition to this enhancement effect, the response pattern across the target force

347 amplitudes was also modified by the use of visual instructions. Improved loudness

348 discrimination was found in the case of the smaller target force in both Motor and

349 Non-Motor conditions in Experiment 1, while discrimination performance was not 350 significantly different among the target force amplitudes in Experiment 2. The 351 difference between Experiment 1 and 2 was also seen in the reaction time data and in session order analysis. These results suggest that visual representation of the target force 
can influence the relationship between the force generation task and the loudness

354 discrimination.

Auditory processing of self-generated sounds has been frequently investigated in

356 the context of sensory attenuation. Previous studies demonstrated attenuated responses

357 to self-generated stimuli compared with otherwise identical sensory stimuli perceived in

358 a passive manner (Blakemore et al. 1998; Martikainen et al. 2004; Aliu et al. 2009;

359 Baess et al. 2009; Horváth 2014; Timm et al. 2014). In these studies, it was

360 hypothesized that the processing of self-generated sounds can be attenuated to

361 distinguish between self-generated and externally generated sounds. These attenuated

responses have been frequently considered as a reduction in sensitivity of sensory

363 processing, but rather indicate an increase in sensitivity (e.g., Eliades and Wang 2003).

364 In the auditory-motor interaction, the previous studies showed that motor commands and corresponding somatosensory inputs can facilitate auditory processing (Reznik et al., 2014; Morillon et al, 2015). Myers et al. (2020) suggested that sensorimotor integration facilitates auditory discrimination. Our findings are consistent with the facilitation of auditory discrimination with motor execution; however, the mechanisms responsible for this relationship may change depending on the context of the auditory-motor interaction tasks. Indeed, context dependency has already been reported by Reznik et al. (2015). 
371 When different auditory contexts were tested using sounds proximal to and larger than

372 the threshold level, the loudness perception changed depending on the sound

373 characteristics, even though the sound stimuli were produced with the same motor task.

374 In the current task involving the force sensor being pressed with the index finger,

375 the specific force amplitude was achieved by adjusting a moving cursor to the visual

376 target. Neszmélyi and Horváth (2017) showed an attenuation effect using a similar type

377 of force generation task. However, their task was to go over a specific threshold level,

378 while our task required the participants to reach a specific force amplitude without

379 exceeding a certain limit. This constraint necessitated more precise control of the force

380 amplitude, which may be key to inducing a facilitatory effect in auditory-motor

381 interactions.

382 It is possible that the prediction of sound timing and the difference in attention level

383 play a role in the changes in discrimination performance between Motor and Non-Motor

384 conditions. As for the stimulus presentation, the participants could easily predict the

385 timing of sound presentation in Motor condition, since the sound was caused by the

386 participants' motor execution. However, the sound timing could also be predicted in

387 Non-Motor condition by observing the moving cursor reaching the target line. Given

that motor prediction and visual prediction are similar (Kaiser et al. 2018, see Weiss and 
389 Schütz-Bosbach, 2012), a prediction mechanism would contribute similarly to both 390 Motor and Non-Motor conditions. Attentional factors might also change depending on 391 the involvement of the force generation task. In general, imposing an additional task 392 while performing a main task disturbs the performance of the main task. In the current 393 experiments, it was expected that the participants would display greater attention to the 394 auditory task in Non-Motor condition. This is consistent with the participants' 395 impressions in the post-experiment interviews. Most of the participants reported that 396 they felt it easier to focus on listening to the sound stimulus in Non-Motor condition 397 than in Motor condition. Despite this, the discrimination performance was better in 398 Motor condition. Thus, the attentional factor may not be involved in the current change 399 in loudness discrimination. This idea may also be supported by the inconsistent result of 400 reaction time between the two experiments. A difference between Motor and Non-Motor 401 conditions was found in Experiment 1, but not in Experiment 2, suggesting that 402 attentional level between Motor and Non-Motor condition may be different in the two 403 experiments. Thus, the differences in prediction and attention between Motor and 404 Non-Motor conditions cannot explain the improvement in loudness discrimination in 405 the force generation task. 
407 fine controls required for performing a motor task, due to interactions between

408 voluntary command, somatosensory, and audition. Macefield et al. (1996) investigated

409 the adjustment of gripping force by two fingers based on skin sensation signals on the

410 fingertip, and suggested that smaller force generation might require more sensitive

411 sensory input feedback. In the current test, finer somatosensory signals may be required

412 for force generation with $1 \mathrm{~N}$ and $2 \mathrm{~N}$, compared to $4 \mathrm{~N}$. Despite this expectation, we

413 did not find any reliable interaction between motor factor and force factor in either

414 Experiment 1 or 2 . In the current context, the loudness perception may not be changed

415 according to the amplitude of the produced force.

416 The main limitation of the present study was that the visual presentation was used

417 for the instruction of the target force amplitude. It was difficult to completely eliminate

418 additional information concerning the differences across the tasks. In Experiment 1, the

419 three target forces were represented by displaying the visual target at different heights.

420 In Experiment 2, while we removed the difference in height, the velocity of the moving

421 cursor varied depending on the target force. The difference in visual instruction induced

422 changes in loudness discrimination. In Experiment 1, the smaller force amplitude

423 (which coincides with the lower height of the visual target) led to improved 
424 discrimination performance. This tendency was not observed in Experiment 2, in which

425 the loudness discrimination was similar among the three target force amplitudes. This

426 difference in visual instruction might also affect reaction time since the result of

427 reaction time was not consistent in the two experiments. The participants responded

428 quicker in Motor condition in Experiment 2, but their responses were not different

429 between Motor and Non-Motor condition in Experiment 1. This suggests that visual

430 instruction or presentation affects the overall performance of the loudness

431 discrimination task. Note that the current visual instructions did not affect the

432 achievement of the force generation task because all participants were able to produce

433 the sound successfully without overshooting a predetermined range of task force

434 amplitude. Regardless of the difference in visual instruction, we found improved

435 loudness discrimination for the self-generated sounds compared to the externally

436 generated sounds. Since the effects of visual instruction were consistent in both Motor

437 and Non-Motor conditions, we concluded that this visual effect can be a separate effect

438 from the current motor effect in auditory perception. We did not pursue this visual effect

439 further in the current study because our purpose is to investigate the motor effect on

440 auditory perception alone. 
442 procedure, we carried out at least one Motor condition before Non-Motor condition in

443 order to replay the visual presentation of Motor condition in Non-Motor condition. This

444 limitation of session order may have caused the current results in loudness

445 discrimination. Considering this order effect, we carried out two session orders

446 (alternated pattern and separated pattern). The main difference between these two

447 session orders was that the 2 nd session and 3 rd sessions were switched. The separated

448 pattern may thereby induce a larger effect than the alternated pattern. Three-way

449 ANOVA with session order (alternated pattern and separated pattern) as a factor showed

450 this tendency in Experiment 1 but not in Experiment 2. This inconsistent result of

451 session order between the two experiments suggests that session order may not be

452 related to the main finding, that is, the improvement of loudness discrimination ability

453 between Motor and Non-Motor conditions was consistently induced in two experiments.

454 To conclude, the current findings support the hypothesis that during 455 sound-producing actions, motor executions modify sensory processing. The 456 auditory-motor interaction required to produce a fixed amount of force might be related 457 to discrimination of auditory consequences by our own movements, such as speaking and playing a musical instrument. 
460 Aliu SO, Houde JF, Nagarajan SS (2009) Motor-induced Suppression of the Auditory Cortex. J. CognNeurosci21:791-802. doi: 10.1162/jocn.2009.21055

462

Baess P, Horváth J, Jacobsen T, Schröger E (2011) Selective suppression of self-initiated sounds in an auditory stream: An ERP study. Psychophysiology 48:1276-1283. doi: 10.1111/j.1469-8986.2011.01196.x

Baess P, Widmann A, Roye A, et al (2009) Attenuated human auditory middle latency response and evoked $40-\mathrm{Hz}$ response to self-initiated sounds. Eur J Neurosci29:1514-1521. doi: 10.1111/j.1460-9568.2009.06683.x

Blakemore S-J, Wolpert DM, Frith CD (1998) Central cancellation of self-produced tickle sensation. Nat Neurosci 1:635-640. doi: 10.1038/2870

Brainard DH (1997) The Psychophysics Toolbox. Spatial Vis 10:433-436. doi: $10.1163 / 156856897 X 00357$

Christensen MS, Lundbye-Jensen J, Geertsen SS, et al (2007) Premotor cortex modulates somatosensory cortex during voluntary movements without proprioceptive feedback. Nat Neurosci 10:417-419. doi: 10.1038/nn1873

Dogge M, Hofman D, Custers R, Aarts H (2019) Exploring the role of motor and non-motor predictive mechanisms in sensory attenuation: Perceptual and 

neurophysiological findings. Neuropsychologia 124:216-225. doi: 10.1016/j.neuropsychologia.2018.12.007

Eliades SJ, Wang X (2003) Sensory-Motor Interaction in the Primate Auditory Cortex During Self-Initiated Vocalizations. Journal of Neurophysiology 89:2194-2207. doi: $10.1152 /$ jn. 00627.2002

Guenther FH, Hampson M, Johnson D (1998) A theoretical investigation of reference frames for the planning of speech movements. Psychol Rev 105:611-633. doi: 10.1037/0033-295X.105.4.611-633

Hickok G, Poeppel D (2007) The cortical organization of speech processing. Nat Rev Neurosci 8:393-402. doi: 10.1038/nrn2113

Horváth J (2014) The role of mechanical impact in action-related auditory attenuation. Cogn Affect BehavNeurosci 14:1392-1406. doi: 10.3758/s13415-014-0283-x

Houde JF (1998) Sensorimotor Adaptation in Speech Production. Science 279:1213-1216. doi: 10.1126/science.279.5354.1213

Hughes G, Desantis A, Waszak F (2013) Attenuation of auditory N1 results from identity-specific action-effect prediction. Eur J Neurosci 37:1152-1158. doi: 10.1111/ejn. 12120 
494

495

496

497

498

499

500

501

502

503

504

505

506

507

508

509

510

511

Kaiser J, Schütz-Bosbach S (2018) Sensory attenuation of self-produced signals does not rely on self-specific motor predictions. Eur J Neurosci 47:1303-1310. doi: 10.1111/ejn.13931

Kinoshita H, Furuya S, Aoki T, Altenmüller E (2007) Loudness control in pianists as exemplified in keystroke force measurements on different touches. The Journal of the Acoustical Society of America 121:2959-2969. doi: 10.1121/1.2717493

Kunde W (2001) Response-effect compatibility in manual choice reaction tasks. J Exp Psychol Hum Percept Perform 27:387-394. doi: 10.1037//0096-1523.27.2.387

Liberman AM, Cooper FS, Shankweiler DP, Studdert-Kennedy M (1967) Perception of the speech code. Psychol Rev 74:431-461. doi: 10.1037/h0020279

Macefield VG, Häger-Ross C, Johansson RS (1996) Control of grip force during restraint of an object held between finger and thumb: responses of cutaneous afferents from the digits. Exp Brain Res 108:155-171. doi: 10.1007/BF00242913

Mario K, David B, Denis P (2007) What's new in Psychtoolbox-3?

Martikainen MH (2004) Suppressed Responses to Self-Triggered Sounds in the Human Auditory Cortex. Cereb Cortex 15:299-302. doi: 10.1093/cercor/bhh131

Meister IG, Wilson SM, Deblieck C, et al (2007) The Essential Role of Premotor Cortex in Speech Perception. Curr Biol 17:1692-1696. doi: 10.1016/j.cub.2007.08.064 
512 Mochida T, Kimura T, Hiroya S, et al (2013) Speech misperception: speaking and seeing interfere differently with hearing. PLoS ONE 8:e68619. doi: 10.1371/journal.pone.0068619

515

Morillon B, Hackett TA, Kajikawa Y, Schroeder CE (2015) Predictive motor control of sensory dynamics in auditory active sensing. CurrOpin in Neurobiol 31:230-238. doi: 10.1016/j.conb.2014.12.005

Myers JC, Mock JR, Golob EJ (2020) Sensorimotor Integration Can Enhance Auditory Perception. Sci Rep 10:1-13. doi: 10.1038/s41598-020-58447-z

Neszmélyi B, Horváth J (2017) Consequences matter: Self-induced tones are used as feedback to optimize tone-eliciting actions: Self-induced tones used as feedback for actions. Psychophysiology 54:904-915. doi: 10.1111/psyp.12845

Niziolek CA, Nagarajan SS, Houde JF (2013) What Does Motor Efference Copy Represent? Evidence from Speech Production. J Neurosci 33:16110-16116. doi: 10.1523/JNEUROSCI.2137-13.2013

Pelli DG (1997) The VideoToolbox software for visual psychophysics: transforming numbers into movies. Spat Vis 10:437-442. doi: 10.1163/156856897X00366

Pfordresher PQ, Palmer C (2006) Effects of hearing the past, present, or future during music performance. Percept Psychophys68:362-376. doi: 10.3758/BF03193683 
530 Reznik D, Henkin Y, Schadel N, Mukamel R (2014) Lateralized enhancement of auditory cortex activity and increased sensitivity to self-generated sounds. Nat Commun 5:1-11. doi: 10.1038/ncomms5059

Reznik D, Henkin Y, Levy O, Mukamel R (2015) Perceived Loudness of Self-Generated Sounds Is Differentially Modified by Expected Sound Intensity. PLoS ONE 10:e0127651. doi: 10.1371/journal.pone.0127651

Sams M, Möttönen R, Sihvonen T (2005) Seeing and hearing others and oneself talk.

Sato A (2008) Action observation modulates auditory perception of the consequence of others' actions. ConsciousCogn 17:1219-1227. doi: Cogn Brain Res 23:429-435. doi: 10.1016/j.cogbrainres.2004.11.006

Sato A (2008) Action observation modulates auditory perception of the consequence of

10.1016/j.concog.2008.01.003

Schneider DM, Nelson A, Mooney R (2014) A synaptic and circuit basis for corollary

Tian X (2010) Mental imagery of speech and movement implicates the dynamics of internal forward models. Front Psychol 1:.doi: 10.3389/fpsyg.2010.00166 Attenuation of Brain Responses to Self-initiated Sounds. J CognNeurosci 
548 Weiss C, Herwig A, Schütz-Bosbach S (2011) The self in action effects: Selective attenuation of self-generated sounds. Cognition 121:207-218. doi:

550 10.1016/j.cognition.2011.06.011

551

552

553

554

555

556

557

558

559

560 doi: $10.1038 / \mathrm{nrn} 2152$ doi: $10.1038 / \mathrm{nn} 2152$

Weiss C, Schütz-Bosbach S (2012) Vicarious action preparation does not result in sensory attenuation of auditory action effects. Conscious and Cogn 21:1654-1661. doi: $10.1016 /$ j.concog.2012.08.010

Wolpert DM, Flanagan JR (2001) Motor prediction. Curr Biol 11:R729-R732. doi: $10.1016 / \mathrm{S} 0960-9822(01) 00432-8$

Wolpert DM, Ghahramani Z, Jordan MI (1995) An internal model for sensorimotor integration. Science 269:1880-1882. doi: 10.1126/science.7569931

Zatorre RJ, Chen JL, Penhune VB (2007) When the brain plays music: auditory-motor interactions in music perception and production. Nat Rev Neurosci 8:547-558.

561 


\section{Figure Legends}

563

564

565

566

567

568

569

570

571

572

573

574

575

576

577

578

579

Fig. 1. Experimental procedure. A) Temporal patterns of auditory stimulation (top) and trajectory of finger force generation with three different target amplitudes (bottom). B) Examples of visual presentation in Experiment 1. The cross mark represents the cursor of the force amplitude. The two horizontal lines represent the target amplitude (bottom line) and upper limit (top line). C) Example of visual presentation in Experiment 2 as in B.

Fig. 2. Mean values of A) just-noticeable difference (JND) and B) point of subjective equality (PSE) in Experiment 1. The solid line with filled circles represents Motor condition, and the dashed line with open circles represents Non-Motor condition. The small dots represent responses in each individual participant. Error bars show the standard error among the participants. PSE is represented as a value relative to $65 \mathrm{~dB}$.

Fig. 3. A) Mean values of A) just-noticeable difference (JND) and B) point of subjective equality (PSE) in Experiment 2. The solid line with filled circles represents Motor condition, and the dashed line with open circles represents Non-Motor condition. 
583

584

585 
A

Tone Stimuli

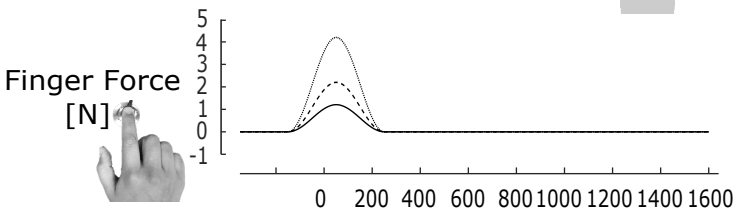

Time [ms]

B

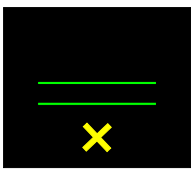

$1 \mathrm{~N}$

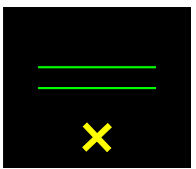

$1 \mathrm{~N}$

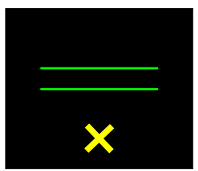

$2 \mathrm{~N}$

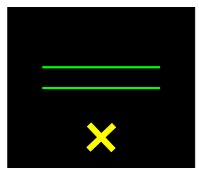

$2 \mathrm{~N}$

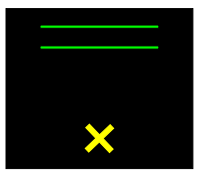

$4 \mathrm{~N}$

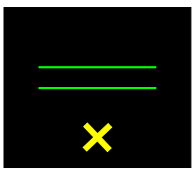

$4 \mathrm{~N}$ 
A

B
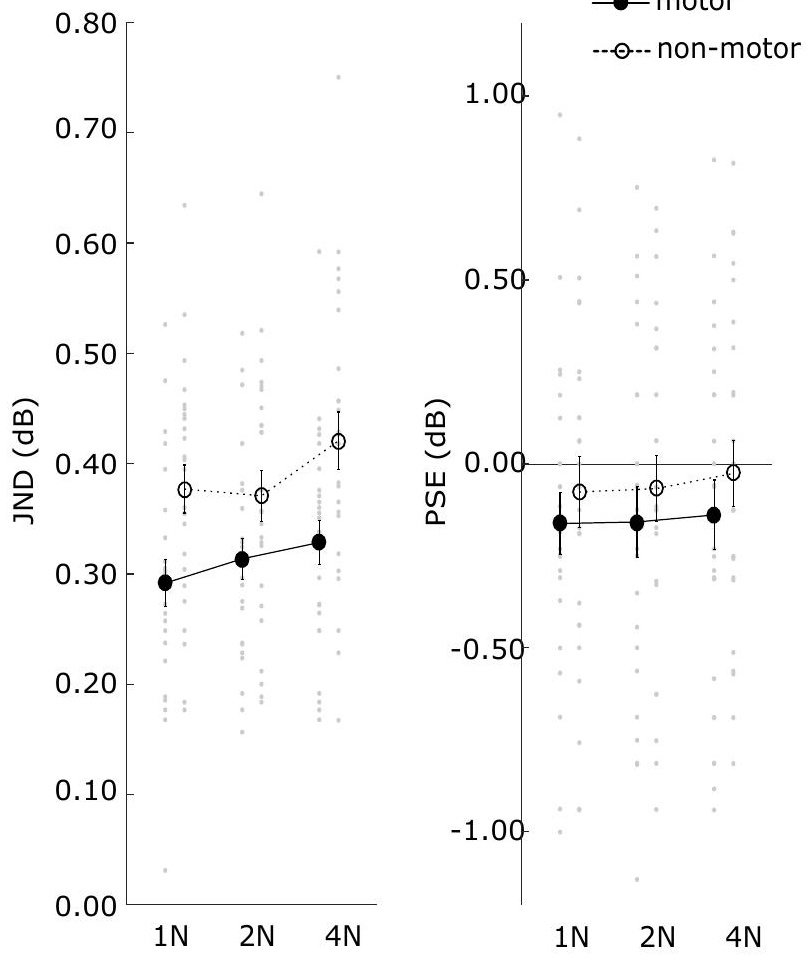
A

B

- motor

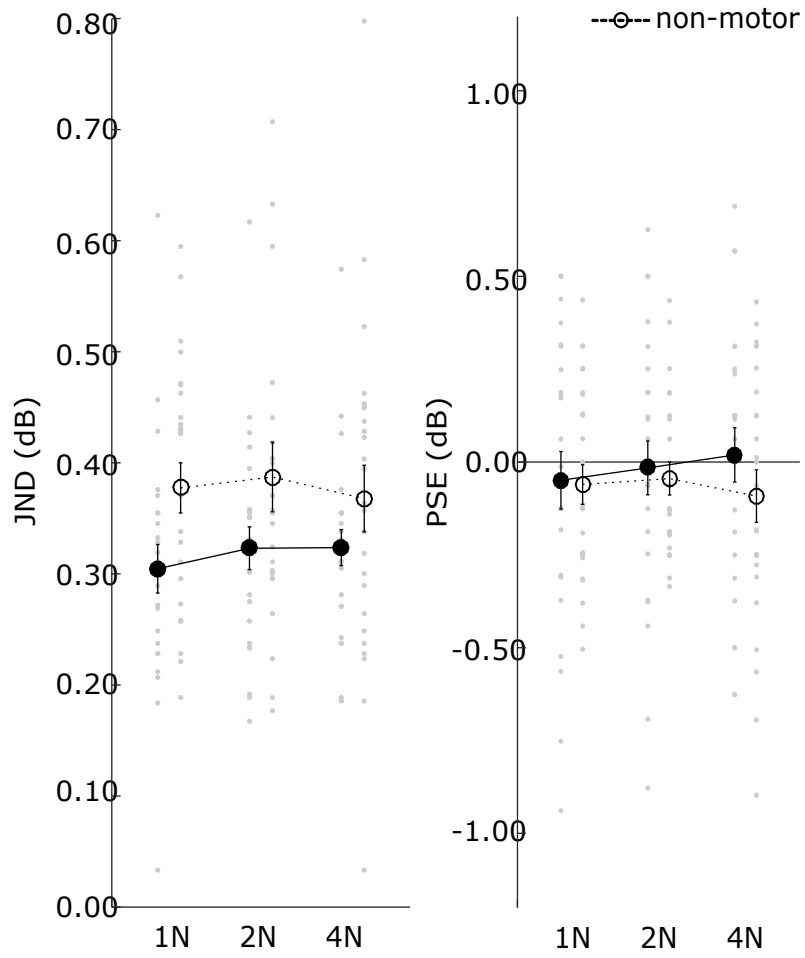

